\title{
Thyroid cancer diagnosis in the era of precision imaging
}

\author{
Kimberley-Jane Bonjoc ${ }^{1}$, Hannah Young ${ }^{1}$, Susanne Warner ${ }^{2}$, Thomas Gernon ${ }^{2}$, Ellie Maghami ${ }^{2}$, \\ Ammar Chaudhry ${ }^{3}$
}

${ }^{1}$ Department of Imaging Administration, City of Hope National Medical Center, Duarte, CA, USA; ${ }^{2}$ Department of Surgery, City of Hope National Medical Center, Duarte, CA, USA; ${ }^{3}$ Department of Diagnostic Radiology, City of Hope National Medical Center, Duarte, CA, USA

Contributions: (I) Conception and Design: KJ Bonjoc, H Young, E Maghami, A Chaudhry; (II) Administrative support: None; (III) Provision of study materials or patients: E Maghami; (IV) Collection and assembly of data: None; (V) Data analysis and interpretation: None; (VI) Manuscript writing: All authors; (VII) Final approval of manuscript: All authors.

Correspondence to: Ammar Chaudhry. 1500 E. Duarte Rd., Duarte, CA, 91010, USA. Email: achaudhry@coh.org.

\begin{abstract}
Thyroid cancer affects $1.3 \%$ of the population with increasing rates of incidence over the last decade (approximately $2 \%$ per year). Although the overall prognosis is good in the differentiated subtypes, there has been a slow but steady increase in rate of deaths associated with thyroid cancer (approximately $0.7 \%$ per year over the last decade). Thyroid cancer is usually detected when: (I) patients feel a lump in the neck; (II) a routine clinical exam is performed; (III) an incidental thyroid nodule is identified on diagnostic imaging (e.g., CT neck or chest, carotid ultrasound, PET scan acquired for non-thyroid pathology). Identification of suspicious thyroid nodules results in further diagnostic work-up including laboratory assessment, further imaging, and biopsy. Accurate diagnosis is required for clinical staging and optimal patient treatment design. In this review, we aim to discuss utility of various imaging modalities and their role in thyroid cancer diagnosis and management. Additionally, we aim to highlight emerging diagnostic techniques that aim to improve diagnostic specificity and accuracy in thyroid cancer, thus paving way for precision medicine.
\end{abstract}

Keywords: Positron emission tomography/computed tomography (PET/CT); ultrasound; magnetic resonance imaging (MRI); thyroid; cancer

Submitted Jul 29, 2019. Accepted for publication Aug 21, 2019.

doi: $10.21037 /$ jtd.2019.08.37

View this article at: http://dx.doi.org/10.21037/jtd.2019.08.37

\section{Epidemiology}

Thyroid cancer is the most common endocrine neoplasm and is the 12 th most common cancer among all cancer types (1). The median 5-year survival rate for thyroid cancer was $98 \%$ between 2009 and 2015; however, prognosis is poor in more aggressive subtypes. For example, anaplastic thyroid cancer (ATC) accounts for less than $1 \%$ of all thyroid cancers, but the median survival is three to seven months and the 1 -year survival rate is $10-20 \%(1,2)$. The incidence of thyroid cancer is almost double now than it was in 2000, accounting for $2.1 \%$ of cancer diagnoses $(3,4)$. Between 1974 and 2013 , the mean age of thyroid cancer diagnosis was $48 \pm 16$. Women are more commonly affected than men, accounting for $75 \%$ of the diagnoses (5). There is increased incidence of thyroid cancer in the geriatric population who also have a worse prognosis from thyroid cancer due to increased treatment related morbidity and mortality (3). Due to the high recurrence rate in thyroid cancer $(20 \%)$, the ability to distinguish recurrence from treatment related changes are key to improving survival (6).

\section{Thyroid cancer subtypes}

There are four major subtypes of thyroid cancer: papillary (PTC), follicular (FTC), anaplastic (ATC), and medullary thyroid cancer (MTC) (1). Less common subtypes include: Hurthle cell carcinoma (HTC) and poorly differentiated thyroid cancer (PDTC). PTC, FTC, and HTC are well differentiated subtypes; accounting for approximately $95 \%$ of thyroid cancers (1). These subtypes generally have a better prognosis than PDTC and ATC subtypes. PTC 

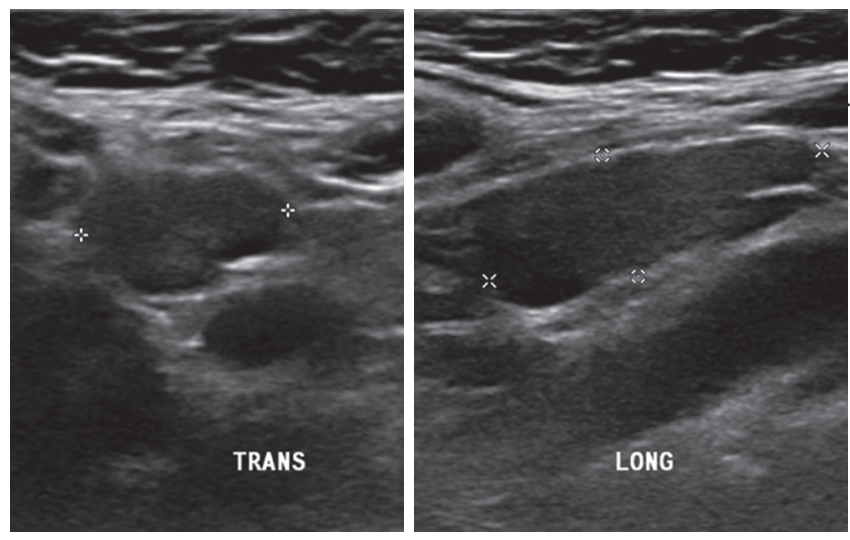

Figure 1 A 60-year-old female's transverse and longitudinal ultrasound presents multiple micropapillary carcinomas with microcalcification and increased vascularity.

integral to the diagnostic work-up and treatment strategies.

\section{Genetics and risk factors}

The most common mutations associated with thyroid cancers are in the BRAF and RET genes, and PAX8/PPAR $\gamma$ genes (7). These mutations alter the MAPK and PI3KAKT pathways. Mutation in the BRAF gene occurs in $40-45 \%$ of de novo PTC while patients with prior radiation exposure most commonly have RET mutation (7). The most common mutation in FTC (40-50\%) and PDTC $(20-40 \%)$ is in the RAS gene (7). A mutation in the TP53 gene occurs in $50-80 \%$ of ATC (7), and is generally not seen in well-differentiated carcinomas, such as PTC and FTC. MTC is unique because the occurrence of the RAS mutation is greater than $95 \%$ in the familial forms, but this mutation is only present in about $25 \%$ of sporadic forms (7). Identification of genetic mutations is essential to diagnosing and staging thyroid cancer in the era of precision medicine with emergence of targeted therapies. ThyroSeqv $2^{\mathrm{TM}}$ is a next generation sequencing panel that is be used to diagnose neoplasms that were classified as indeterminate cytology (8). Chudova et al. have utilized algorithms to differentiate between benign and malignant tumor using gene expression profiles (9). Wylie et al. utilized miRNA gene expression profiles and somatic gene alterations to develop molecular classifications of thyroid cancer (10). Sanler et al. analyzed the connection between germline mutations in DNA repair genes and risk of thyroid cancer (11). Next generation sequencing (NGS) technologies have allowed for more rapid, detailed, and accurate identification of molecular abnormalities in thyroid cancers; making them a routine part of practice at academic institutions. As precision medicine grows, diagnostic and treatment strategies revolving around genetic mutations will probably become standard practice in both academic and conventional practices.

\section{Thyroid cancer diagnosis: precision imaging}

\section{Ultrasound}

Ultrasound is the initial modality of choice to evaluate thyroid nodules and distinguish benign from neoplastic nodules. Ultrasound is cost-effective, readily available, and does not expose patients to radiation. Thyroid nodules with suspicious features are often biopsied using ultrasoundguided fine needle aspiration (FNA). Ultrasound is not only useful in detecting primary neoplasm, but it can evaluate locoregional lymph nodes for nodal metastasis. Ultrasound guidance is also frequently used for operative planning to ensure suspicious nodule(s) and lymph nodes have been resected (12). In diagnostic ultrasounds (Figure 1), thyroid nodules are characterized based on: size, margins, eccentric location of solid portion, hypoechogenicity, microcalcification, irregular shape, and if the tumor is taller than it is wide (1).

Features extracted from this criterion help distinguish benign from malignant thyroid nodules. For example, irregular margins and microcalcifications (Figure 2) are associated with malignant nodules while small subcentimeter spongiform avascular nodules are considered benign (12). The Bethesda System for Reporting Thyroid Cytopathology (BRSTC) is a diagnostic classification system based on FNA cytology. This system has six diagnostic categories: non-diagnostic or unsatisfactory, benign, atypia of undetermined significance or follicular lesion of undetermined significance, follicular neoplasm or suspicious for a follicular neoplasm, suspicious for malignancy, and malignant (13). Approximately $20 \%$ of FNA are classified as atypia of undetermined significance or follicular lesion of undetermined significance (14).

The American College of Radiologists (ACR) uses a thyroid cancer diagnostic management system called the Thyroid Imaging Reporting and Data System (TIRADS). TI-RADS is increasingly used by radiologists and other healthcare practitioners to plan management of thyroid tumors, which may include FNA cytology, surgery, 

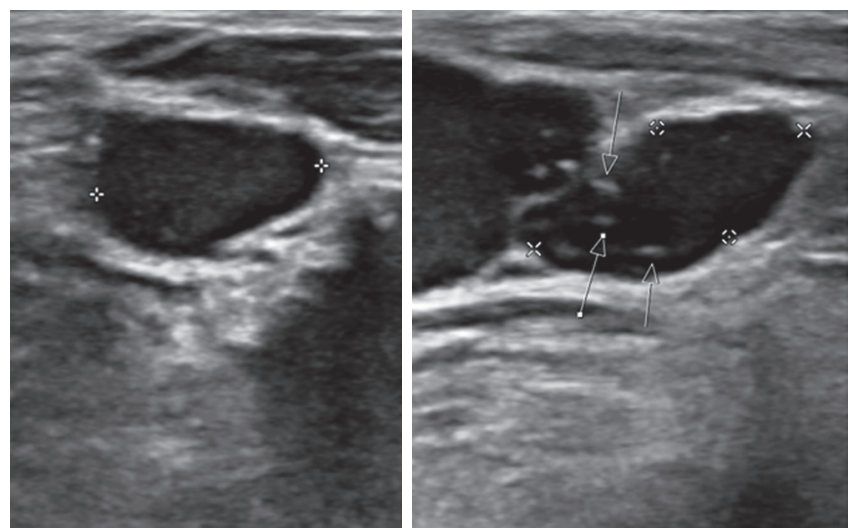

Figure 2 A 82 -year-old female diagnosed with metastatic medullary and classical papillary thyroid carcinoma. Transverse (left) and longitudinal (right) ultrasound displays thyroid lesion with microcalcifications indicated by white arrows.

and administration of radiotherapy. TI-RADS criteria is based on composition, echogenicity, shape/morphology margins (15). Each criterion is scored based on risk levels range from benign to highly suspicious designated by TR1 and TR5, respectively (15). The criteria, scoring system, classification and recommendations based on classifications that are shown in Tables 1,2 $(16,17)$. These risk levels help to determine management of thyroid tumors. Wu et al. compared the efficacy of the BSRTC system to the TI-RADS classification and BRAF mutation diagnosis. Previously, the BRAF mutation was found to have a low sensitivity, but almost $100 \%$ specificity (14). The BRAF mutation showed similar clinical value to TI-RADS in the nodules classified as BSRTC III/V. As BRAF mutation is found in $40-45 \%$ of PTC (7), and PTC is the most common subtype; TI-RAD derived diagnosis of thyroid nodules can provide a non-invasive radiogenomic approach that can potentially predict underlying common genomic alterations in these thyroid neoplasms.

Although FNA cytology can detect half of MTC's, additional imaging tests are necessary to accurately diagnose MTC. A study was done to assess the usefulness of the ACR TI-RADS versus a modified TI-RADS to diagnose MTC (15). The TI-RADS criteria were analyzed based on sensitivity, specificity, negative predictive value, accuracy, and Youden index. Zhu et al. concluded that the modified TI-RADS was a better framework for diagnosing MTC's than the ACR TI-RADS (15).

There are additional ultrasound-based classification systems from the British Thyroid Association (BTA),
American Thyroid Association (ATA), and American Association of Clinical Endocrinologists (AACE) (18). These classifications are used based on locoregional practice guidelines that are impacted by geographic location of practice and training in the respective experts of the thyroid cancer disease terms. For example, in the United States, radiologists mostly adhere to practice guidelines put forth by the American College of Radiology (ACR) and American Board of Radiology (ABR); thus resulting in rapid adaptation of TI-RADS in clinical practice. It should be noted, however, that ultrasounds have limitations and are not always predictive for malignancy. Ram et al. showed that sensitivity and specificity of ultrasound in predicting malignancy were $80 \%$ and $68 \%$, respectively (19). However, Brito et al. in their meta-analysis showed there are multiple studies that have quoted variable levels of diagnostic accuracy of ultrasound and the degree of accuracy does depend on the experience of the physician interpreting the ultrasound (20). As such, in patients with high index of suspicion for primary or recurrent thyroid cancer, other imaging modalities [single-photon emission computed tomography (SPECT), computed tomography (CT), magnetic resonance imaging (MRI), etc.] should be used to rule out disease.

\section{SPECT}

${ }^{123}$ Iodine SPECT scans is an important diagnostic tool used in clinical practice for work-up of indeterminate or suspicious thyroid nodules as well as staging of thyroid cancer for over six decades (21). Sodium-iodide symporters (NIS) located on the basolateral plasma membrane of thyrocytes leads to increased affinity for iodine and this physiologic mechanism is leveraged in radioiodine imaging and therapy (21). Whole-body scintigraphy (WBS) is commonly performed with 123Iodine to evaluate whether a suspicious or indeterminate thyroid nodule is "hot" (increased iodine uptake) or "cold" (diminished thyroid uptake) (22). Radioiodine scans are particularly useful in differentiated subtypes of thyroid cancer due to preserved NIS allowing for preferential increased accumulation of radioactive iodine in the neoplasm compared to normal thyroid tissue (Figure 3). As thyroid neoplasms dedifferentiate, they lose the NIS rendering abilities these lesions occult on radioiodine SPECT; an important falsenegative to be aware of. In patients post thyroidectomy and/or lymph node dissection, radioiodine scan can help identify residual thyroid tissue and/or identify additional 
Table 1 Presents the five categories (TR1-TR5), the scoring for each category, the classification, and recommendations for each category defined by the Thyroid Imaging, Reporting, and Data Sysem (TIRADS) developed by the American College of Radiologists (ACR). Table was adapted from information from Tessler et al. and Grant et al. $(16,17)$

\begin{tabular}{|c|c|c|c|c|c|}
\hline Category & TR1 & TR2 & TR3 & TR4 & TR5 \\
\hline Classification & Benign & Not suspicious & Mildly suspicious & Moderately suspicious & Highly suspicious \\
\hline Recommendations & No FNA required & No FNA required & $\begin{array}{l}\geq 1.5 \mathrm{~cm} \text { follow up, } \\
\geq 2.5 \mathrm{~cm} \text { FNA; } \\
\text { follow up: } 1,3,5 \text { years }\end{array}$ & $\begin{array}{l}\geq 1.0 \mathrm{~cm} \text { follow up, } \\
\geq 1.5 \mathrm{~cm} \text { FNA; } \\
\text { follow up: } 1,3,5 \text { years }\end{array}$ & $\begin{array}{l}\geq 0.5 \mathrm{~cm} \text { follow up, } \\
\geq 1.0 \mathrm{~cm} \text { FNA; annual follow } \\
\text { up for up to } 5 \text { years }\end{array}$ \\
\hline
\end{tabular}

Table 2 Presents the criteria (composition, echogenicity, shape, margin, and echogenic foci), the possible characteristics of each criteria (e.g., cystic or almost completely cystic in the composition criteria), and the scores for each characteristic defined by the Thyroid Imaging, Reporting, and Data System (TIRADS) developed by the American College of Radiologists (ACR). Table was adapted from information from Tessler et al. and Grant et al. $(16,17)$

\begin{tabular}{|c|c|c|}
\hline Feature & Score & Description \\
\hline \multirow[t]{4}{*}{ Composition } & 0 & Cystic or almost completely cystic \\
\hline & 0 & Spongiform \\
\hline & 1 & Mixed cystic and sold \\
\hline & 2 & Solid or almost completely solid \\
\hline \multirow[t]{4}{*}{ Echogenicity } & 0 & Anechoic \\
\hline & 1 & Hyperechoic or isoechoic \\
\hline & 2 & Hypoechoic \\
\hline & 3 & Very hypoechoic \\
\hline \multirow[t]{2}{*}{ Shape } & 0 & Wider-than-tall \\
\hline & 3 & Taller-than-wide \\
\hline \multirow[t]{4}{*}{ Margin } & 0 & Smooth \\
\hline & 0 & III-defined \\
\hline & 2 & Lobulated or irregular \\
\hline & 3 & Extra-thyroidal extension \\
\hline \multirow[t]{4}{*}{ Echogenic foci } & 0 & None or large comet-tail artifacts \\
\hline & 1 & Macrocalcifications \\
\hline & 2 & Peripheral (rim) calcifications \\
\hline & 3 & Punctate echogenic foci \\
\hline
\end{tabular}

sites of metastasis (21). ${ }^{131}$ Iodine $\left({ }^{131} \mathrm{I}\right)$ emits both beta and gamma particles, making it the perfect theranostics agent. ${ }^{131} \mathrm{I}$ is commonly administered post-thyroidectomy with or without nodal dissection for treatment of residual thyroid tissue and for treatment of local/distal metastasis (21). Additionally, due to 131I gamma activity, SPECT can be performed to evaluate response to 131Iodine radioablation. Post-ablation SPECT is usually performed within fiveto-nine days due to improved signal-to-noise ration that increases overall sensitivity for detection of distant metastases (21).

\section{$C T$}

With continual increase in use of CT, unsuspected thyroid nodules are often detected on CT (23). For example, incidental thyroid nodule detection rate on $\mathrm{CT}$ chest ranges from $2-65 \%$ (23). Although majority of these incidental thyroid nodules are benign, $5 \%$ of these nodules are malignant (23). Due to continued increase in utilization of CT with increased detection of thyroid nodules, coupled with a decade long trend of increased incidence of thyroid neoplasm, the ACR put forth recommendations for management of incidental thyroid nodules as referred in Figure 4 (24). Thyroid nodules with suspicious features on $\mathrm{CT}$ are followed-up with ultrasound and may require ultrasound-guided FNA biopsies to determine whether the thyroid lesion is benign or malignant (23). Although CT is not the initial modality of choice for evaluation of primary thyroid cancer, it is useful for staging thyroid cancer in detection of metastasis. In cases where primary thyroid lesion has substernal extension or there is concern for airway compromise, CT is the preferred diagnostic modality (Figure 5).

\section{Positron emission tomography/computed tomography (PET/CT)}

Due to simultaneous acquisition of structural and functional information, PET/CT is widely used for the staging/ 


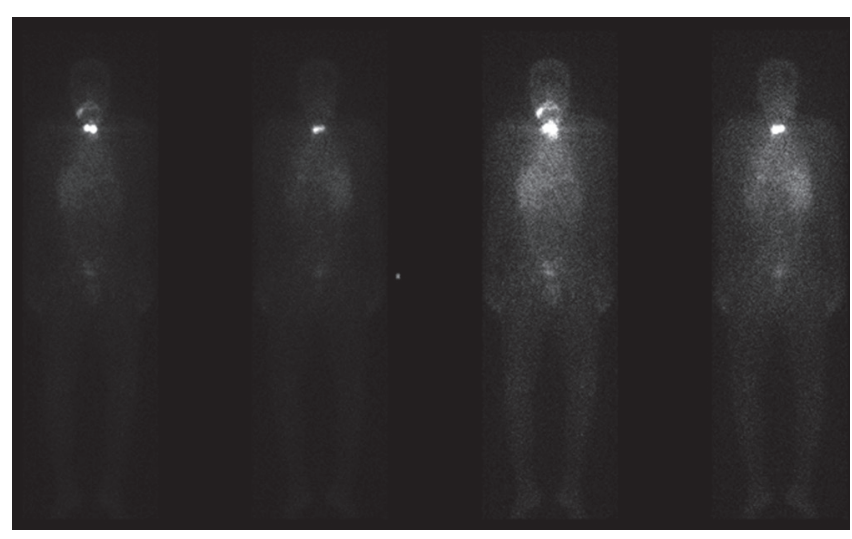

Figure 3 A 39-year-old male patient diagnosed with metastatic papillary thyroid carcinoma. Patient presents 131-Iodine uptake in I-SPECT scan, indicating follicular adenoma. restaging of malignancies (25). As previously mentioned, differentiated thyroid neoplasms have preserved NIS and are adequately assessed on SPECT. De-differentiated or poorly differentiated thyroid neoplasms are occult on radioiodine SPECT due to loss of NIS. However, due to increased metabolic activity in these aggressive neoplasms (Figures 6,7), ${ }^{18}$ Fluorodeoxyglucose (FDG) PET/CT can be used to reliably stage these patients (21). In patients with high levels of thyroglobulin and a negative iodine scan, PET/CT can assess extent of metastatic disease, assist in radiotherapy planning, and can assess patient response to treatment (25). The quantitative measurement of FDG-PET/CT have been shown to have predictive and prognostic value $(25,26)$. As such, FDG $\mathrm{PET} / \mathrm{CT}$ is the preferred modality for restaging patients

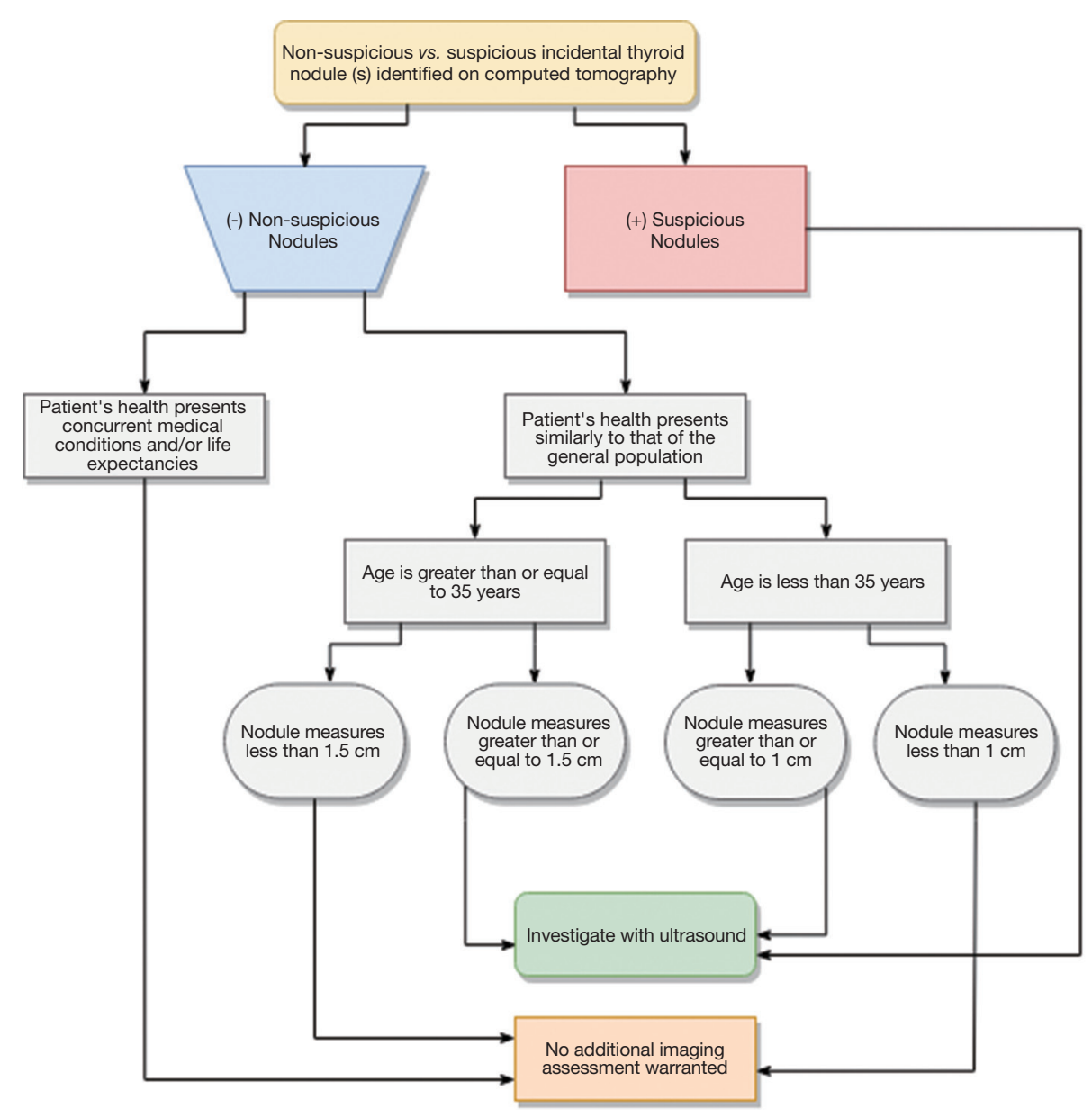

Figure 4 Clinical work-up for incidental thyroids identified on computed tomography (24). 


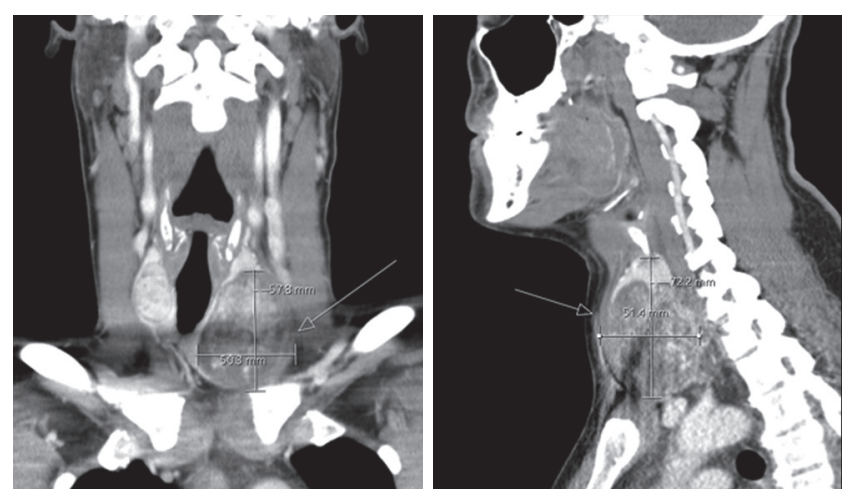

Figure 5 Coronal (left) and sagittal (right) CT of a 39-yearold male patient diagnosed with metastatic papillary thyroid carcinoma. Patient presents a heterogeneously enhancing thyroid nodule that is eroding through the thyroid capsule (white arrows).
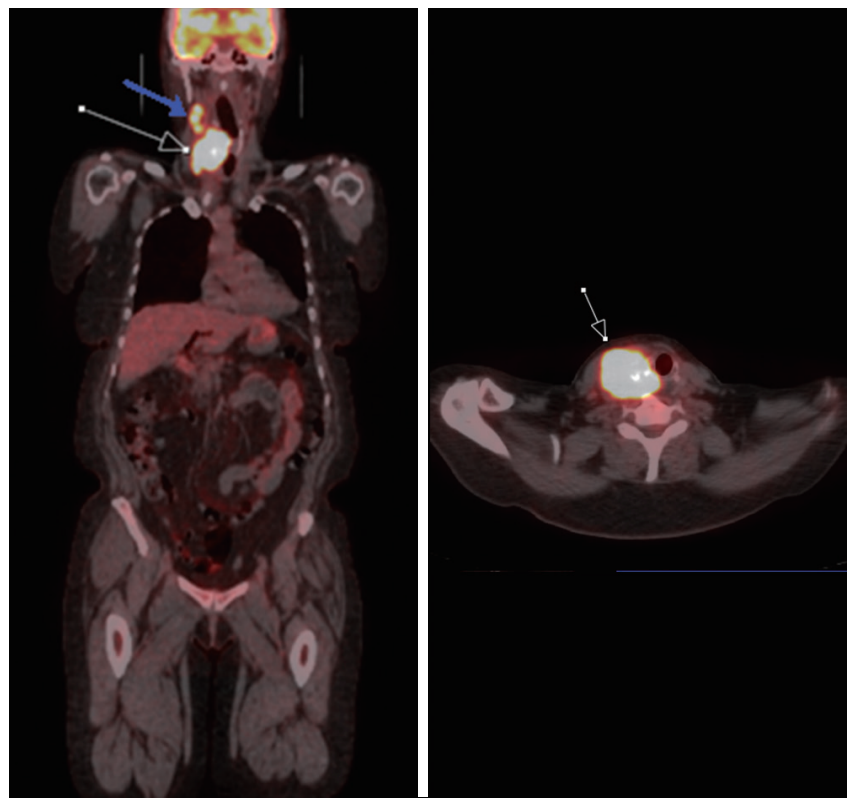

Figure 6 A 66-year-old female diagnosed with poorly differentiated thyroid carcinoma. FDG PET/CT demonstrates increased FDG uptake in the thyroid nodule (white arrow) Additionally, there is FDG avid metastasis to ipsilateral cervical chain lymph node (blue arrow). PET/CT, positron emission tomography/computed tomography; FDG, fluorodeoxyglucose.

with dedifferentiate thyroid neoplasms for whole body assessment. A key limitation of FDG PET/CT is increased rates of false positives in a setting of co-existing infection and/or inflammation. For example, it is often difficult to discriminate between radiation-related inflammation from
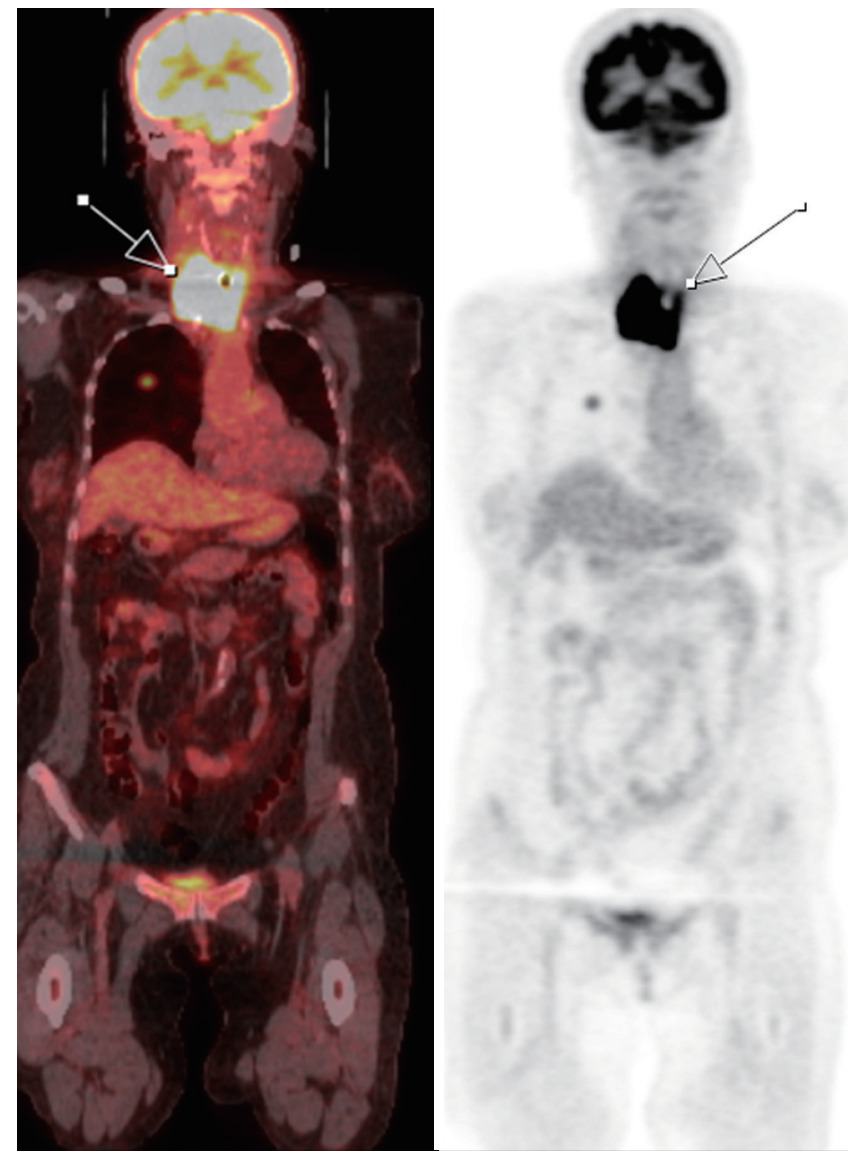

Figure 7 A 74-year-old female diagnosed with anaplastic thyroid carcinoma. PET/CT scan shows increased FDG uptake within an enlarged thyroid with irregular margins (white arrow) encasing the trachea. PET/CT, positron emission tomography/computed tomography; FDG, fluorodeoxyglucose.

residual neoplasm in ATC. Equivocal cases on PET/CT may require further evaluation of other modalities (MRI, PET/MRI) and/or biopsy to improve diagnostic specificity. Another important limitation of whole-body PET/CT is larger slice thickness, which limits assessment of pulmonary micrometastasis. In such instances, dedicated high-resolution CT of the chest is the best diagnostic modality. Furthermore, due to limited soft tissue resolution of PET and CT, if there is concern for CNS and/or spinal metastasis, MRI or PET/ MRI are the preferred diagnostic modalities.

\section{MRI}

Due to cost and limited availability compared to other modalities, MRI is used most frequently in a second line 

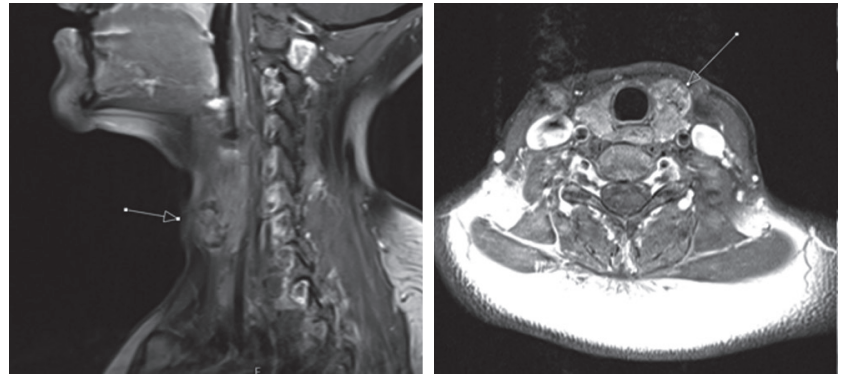

Figure 8 Sagittal (left) and axial (right) T1-post contrast MRI of a 37-year-old female diagnosed with papillary thyroid carcinoma. T1-post-contrast MRI demonstrates heterogeneously enhancing nodule in the anterior aspect of left thyroid lobe (arrows) with wellcircumscribed borders without evidence of ipsilateral adenoapthy or tracheal invasion. MRI, magnetic resonance imaging.

setting for characterization of tumor in suspicious regions. Ultrasound is the modality of choice for initial diagnosis and follow-up assessment in thyroid cancer. In cases where recurrence/relapse is suspected, MRI is utilized due to its superior soft tissue resolution relative to ultrasound, CT, PET/CT, and SPECT (Figure 8). In high risk patients (e.g., incidence of familiar neoplasm, aggressive initial cancer with positive margins, etc.), MRI is the modality of choice to survey for recurrence. Improved contrast and soft tissue resolution on newer MRI techniques provides more precise evaluation of tumor extent and infiltration of surrounding tissues (vessels, nerves, bones, etc.). MRI techniques such as diffusion weighted imaging (DWI), coupled with changes in other sequences (T1- and T2-weighted) can help discriminate between benign and malignant thyroid lesions $(27,28)$. Perfusion MRI, which evaluates degree to tumor vascularity and tumoral blood flow relative to the normal tissue, has also shown to be a helpful technique in discriminating benign and malignant lesions (29). Certain implantable cardiac devices and other metallic implants had been a limitation of MRI; however, due to clinical need for MRI in accurate diagnosis, this has led manufacturers to develop MRI compatible devices. Additionally, there are newer MRI protocols developed by the American College of Radiology and other organizations allowing for safe imaging of patients with implantable devices. One key challenge, which is an active area of research in MRI (30), is overcoming image quality degradation from susceptibility artifact that generates from metals and air. These artifacts are commonly present in the surgical site, limiting evaluation of local disease. Newer surgical techniques and newer MRI sequences are being developed to overcome these limitations (30).

\section{Positron emission tomography/magnetic resonance imaging (PET/MRI)}

PET/MRI is a hybrid modality providing simultaneous acquisition of PET and MRI data. This modality combines the individual strengths of PET and MRI as previously discussed which allow it to overcome the limitation of PET/ CT. For example, Binse et al. showed that PET is more accurate in the diagnosis of the spatial extent of disease, provides better morphologic characterization, especially in the operative bed, and shows improved detection of disease in the brain, bones, and spinal canal. Additionally, the PET detectors in PET/MRI are more sensitive and thus about to detect more lesions than conventional PET/CT detectors (31). Furthermore, in the postoperative and postablative setting, MRI is better than CT at assessing residual and/or recurrent in the treatment region than CT. As the MRI component in PET/MRI does not require radiation, there is overall decreased exposure to ionizing radiation from PET/MRI than PET/CT (31). Recently, in a study conducted by Jentzen et al., the quantitative performance of ${ }^{124} \mathrm{I}$ PET/MRI and ${ }^{124} \mathrm{I}$ PET/CT were compared. The study showed comparable quantitative PET performance between the two aforementioned modalities, further suggesting that PET/MRI can be reliably used for evaluation of thyroid cancer in initial staging and follow-up settings (32).

\section{Theranostics}

Theranostics incorporates diagnostic methods and therapeutics to develop precision medicine techniques to give patients personalized treatments (33). Radiotheranostics is the subset of theranostics that incorporates a radioactive component which can be used for diagnostic imaging and therapy (33). As previously mentioned, one of the first radiotheranostic agent used was radioiodine (131I) for treatment of thyroid cancer. Presence of NIS in differentiated thyroid neoplasms allows the tumors to be both ablated and visualized on ${ }^{131} \mathrm{I}$ WBS of thyroid cancer. Radioconjugated metaiodobenzylguanidine (MIBG) is another commonly used radiotheranostic agent for diagnosis and treatment of a variety of neuroendocrine tumors, including MTC, by using ${ }^{123} \mathrm{I}$ and ${ }^{131} \mathrm{I}$, respectively $(33) .{ }^{68}$ Gallium $\left({ }^{68} \mathrm{Ga}\right.$ ) based SPECT and PET radiotracers have also been used to diagnose and assess treatment response in thyroid cancer (34). Prostate 
specific membrane antigen (PSMA) is not only expressed in prostate cancers, but is also expressed in the endothelial cells of the neovasculature in non-prostate tumors. PSMA antibodies radioconjugated with ${ }^{18} \mathrm{~F}$ and ${ }^{68} \mathrm{Ga}$ and have shown uptake in both FTC and adenomas (35). MTC have increased (92\%) expression of PSMA in the neovasculature, but are not expressed on surface of thyroid tumor cells. This suggests PSMA-based probes may be a novel theranostic agent useful in treating hypervascular thyroid neoplasms (33). ${ }^{18} \mathrm{~F}$-tetra-fluoroborate $\left({ }^{18} \mathrm{~F}\right.$-TFB) is a PET radiotracer that is transported intracellularly via NIS and can be used to image well-differentiated thyroid neoplasms that have preserved NIS (35). The benefit of using ${ }^{18} \mathrm{~F}-\mathrm{TFB}$ over ${ }^{123} \mathrm{I}$ is improved signal to noise ratio which translates into improved image quality. Additional benefit of ${ }^{18} \mathrm{~F}-\mathrm{TFB}$ over ${ }^{123} \mathrm{I}$ is lower radiation dose. Future studies are required comparing superior diagnostic capabilities of ${ }^{18} \mathrm{~F}-\mathrm{TFB}$ over ${ }^{123} \mathrm{I}$.

\section{Response Evaluation Criteria in Solid Tumors (RECIST)}

There are a variety of diagnostic criteria that are being used to analyze image data to predict treatment response. RECIST 1.1, created in 2000 (RECIST 1.0) and then revised in 2009 (RECIST 1.1), is the most commonly used treatment response evaluation criteria that uses morphologic criterion requiring a well-defined measurable lesion (36). Evaluation entails one-dimensional and twodimensional measurements of maximal transverse diameters of target lesions. The interval change in lesion size over time is used to determine treatment response (36). Determining the minimum size of the tumor depends on the assessment method for the selection of both target and non-target lesions. For example, lesions for CT or MRI scans require a minimum of one centimeter in diameter (36). The categories used to describe the responsive morphologies of the solid tumor during treatment for this criterion are as follows: complete response (CR), partial response (PR), progressive disease (PD) [can also be described as disease progression (DP)], and stable disease (SD) (36). CR is described as total tumor disappearance, PR is described as a greater than thirty percent reduction in the tumor's diameter, PD is assessed as a greater than twenty percent increase in the size of the tumor's diameter, and SD is described as having less than a thirty percent reduction in diameter and/or less than a twenty percent increase in the diameter (36).

\section{Immune-related Response Evaluation Criteria in Solid Tumors (ir-RECIST)}

The ir-RECIST is analogous to RECIST 1.1 and evaluates treatment response using tumor size. The key difference between RECIST 1.1 and ir-RECIST is that "ir" allows for inclusion of pseudoprogression (PPD) which is related to immunologic change in the tumor from treatment (e.g., radiation, immunotherapy). Response in ir-RECIST is evaluated as: "ir-CR" is immune-related complete response, "ir-PR" is immune-related Partial Response, "ir-PD" is immune-related Progressive Disease, and "irSD" is immune-related Stable Disease (37). ir-RECIST was developed because, unlike conventional cytotoxic chemotherapeutics, emerging immunotherapeutics were resulting in increased incidence of $\mathrm{PPD}$, which refers to transient increase size of primary tumor bed due to recruitment of inflammatory cells. PD on RECIST 1.1 is usually a terminal criteria for patients on a clinical trial (36). Since RECIST 1.1 does not have a specific criterion for PPD, ir-RECIST was developed to account for PPD, which allowed for patients to continue the clinical trial until definitive PD was established on subsequent evaluations $(36,38)$.

\section{Positron emission tomography evaluation response criteria in solid tumors (PERCIST)}

PERCIST is a criterion based on FDG-PET/CT and uses interval changes in metabolic activity as a signature of treatment response $(39,40)$. Various solid-tumor diseases utilize ${ }^{18} \mathrm{~F}-\mathrm{FDG}$ PET/CT. PERCIST evaluation is most commonly utilized in small-cell lung cancer, esophageal cancer, colorectal cancer, breast cancer, head and neck, and uterus (40-42). Investigators may use PERCIST over RECIST and ir-RECIST in scenarios where there is no "measurable" disease based on size criteria (e.g., infiltrative non-mass-like breast or head/neck cancer) or in scenarios where change is size lags behind change in metabolic activity (e.g., small cell lung cancer).

Basal requirements for this criterion include measurements of the single "hottest" tumor, indicating intense FDG uptake and establishing the most metabolically aggressive site of the tumor, and background area, usually a three-centimeter diameter spherical volume placed on the right side the liver (40). Measuring these aspects establishes a baseline that allows for comparable readings of FDG uptake at different time points (40). Subsequent 

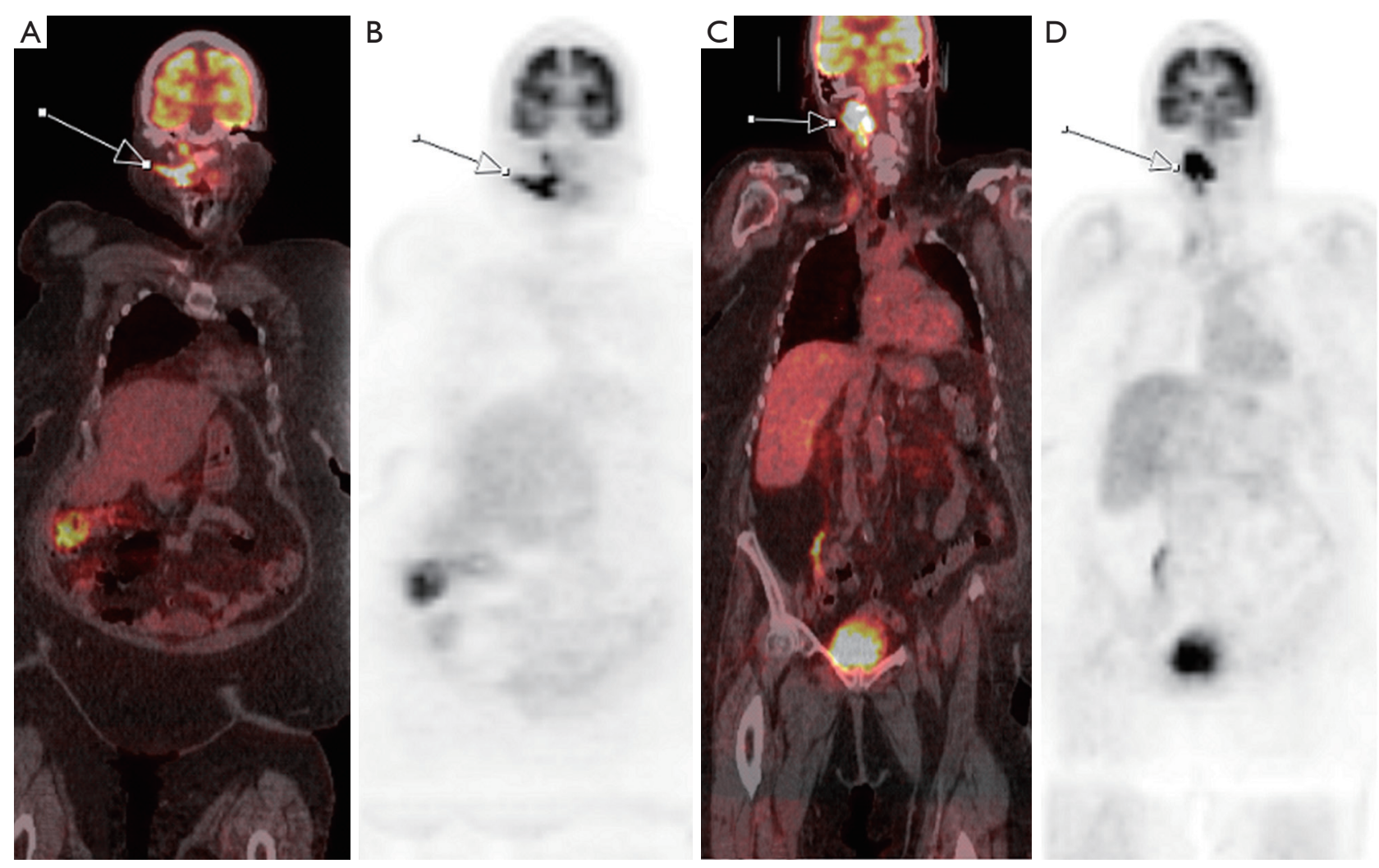

Figure 9 A 79-year-old female diagnosed with metastatic Hurthle cell carcinoma presents radioactive iodine (RAI)-resistance. Initial FDG PET/CT shows increased metabolic activity in the recurrent metastasic right level II/III cervical chain lymph nodes (white arrows) in 2016. Six months after RAI therapy, follow-up FDG PET/CT in 2017 shows increased size and metabolic activity in the right level II/III cervical chain lymph nodes, indicating RAI-resistance. PET/CT, positron emission tomography/computed tomography; FDG, fluorodeoxyglucose; RAI, radioactive iodine.

readings from the initial scan are then compared to the established baseline, thus indicating either an increase or decrease in intensity of FDG uptake (40). Categories for this criterion are described as the following: Complete Metabolic Response (CMR), Partial Metabolic Response (PMR), Progressive Metabolic Disease (PMD), and Stable Metabolic Disease (SMD) (40).

As previously addressed, RECIST 1.1 is a response evaluation criterion that is dependent on morphological changes, while PERCIST allows for the interpretation of the metabolic activity response in the targeted solid tumor (Figure 9).

During the emergence of targeted therapies in the era of precision medicine, some investigators favor PERCIST over RECIST $1.1(39,41)$. The primary reason is that targeted therapies often cause incidents of necrosis and cystic change rather than tumor regression alone. As tumor regression is a key factor in determining a patient's response to treatment in RECIST 1.1 it is not well suited in determining efficacy of targeted therapies in solid tumors $(39,41)$. It is important to note that there are no randomized controlled studies that have shown superiority of RECIST 1.1 over PERCIST, and vice versa. Aras et al. compared RECIST 1.1 and PERCIST that show aspects of response agreement between the criterions that validate the importance of response analyses (43). Although the agreement of tumor responses between RECIST 1.1 and PERCIST were satisfactory, adopting PERCIST over RECIST 1.1 caused a significant shift in the overall response rates to target therapies from $30-35 \%$ to $50-55 \%(39,41) .80 \%$ of patients classified as "SD" using RECIST 1.1 were upgraded to CMR or PMR when adopting PERCIST (39). Given significant implications of response assessment on pivotal clinical trial, large randomized studies are required to establish superiority of one criteria over the other to improve homogeneity of response assessment across disease types. 


\section{Conclusions}

As prevalence and incidence of thyroid cancer increases, accurate timely evaluation of thyroid nodules is needed to improve survival. Imaging is integral to patient diagnosis and follow-up in thyroid cancer, with ultrasound being the primary modality of choice for evaluation of thyroid lesions. Indeterminate and suspicious lesions are either followed up with SPECT or FNA depending on ultrasound findings and managed accordingly. CT has limited role in characterizing intra-thyroidal lesions and is more commonly used to evaluate metastatic disease. MRI has superior soft tissue contrast and resolution can discriminate between benign and malignant nodules. However, due to cost and access, it is most commonly utilized in the post-operative setting in high risk patients to evaluation for residual and/or recurrent disease. For decades, radioiodine has been used as a diagnostic and therapeutic (theranostics) tool because of preserved NIS in well-differentiated thyroid neoplasms. PET/CT and PET/MRI are best suited for evaluation of de-differentiated to poorly differentiated thyroid neoplasms, with emerging studies suggesting PET/MRI possibly being superior to PET/CT for staging of thyroid cancer in initial or follow-up settings. In the era of precision medicine with increasing available FDA approved targeted treatments, significant research is being done for development of novel radiotracers to improve diagnostics specificity of initial tumor as well as improve accuracy of treatment response assessment.

\section{Acknowledgments}

Funding: None.

\section{Footnote}

Provenance and Peer Review: This article was commissioned by the Guest Editor (Ammar Chaudhry) for the series "Role of Precision Imaging in Thoracic Disease" published in Fournal of Thoracic Disease. The article was sent for external peer review organized by the Guest Editor and the editorial office.

Conflicts of Interest: All authors have completed the ICMJE uniform disclosure form (available at http://dx.doi. org/10.21037/jtd.2019.08.37). The series "Role of Precision Imaging in Thoracic Disease" was commissioned by the editorial office without any funding or sponsorship. AC served as the unpaid Guest Editor for the series. The other authors have no other conflicts of interest to declare.

Ethical Statement: The authors are accountable for all aspects of the work in ensuring that questions related to the accuracy or integrity of any part of the work are appropriately investigated and resolved.

Open Access Statement: This is an Open Access article distributed in accordance with the Creative Commons Attribution-NonCommercial-NoDerivs 4.0 International License (CC BY-NC-ND 4.0), which permits the noncommercial replication and distribution of the article with the strict proviso that no changes or edits are made and the original work is properly cited (including links to both the formal publication through the relevant DOI and the license). See: https://creativecommons.org/licenses/by-nc-nd/4.0/.

\section{References}

1. Shore SL. Thyroid Cancer. In: Vinjamuri S, editor. PET/ CT in Thyroid Cancer. Cham: Springer International Publishing; 2018. p. 1-7.

2. Shore SL. Thyroid Cancer Pathology. In: Vinjamuri S, editor. PET/CT in Thyroid Cancer. Cham: Springer International Publishing; 2018. p. 9-13.

3. Haymart MR, Banerjee M, Reyes-Gastelum D, et al. Thyroid Ultrasound and the Increase in Diagnosis of Low-Risk Thyroid Cancer. J Clin Endocrinol Metab 2019;104:785-92.

4. Kitahara CM, Sosa JA. The changing incidence of thyroid cancer. Nat Rev Endocrinol 2016;12:646-53.

5. Lim H, Devesa SS, Sosa JA, et al. Trends in Thyroid Cancer Incidence and Mortality in the United States, 1974-2013. JAMA 2017;317:1338-48.

6. Lubitz CC, Sosa JA. The changing landscape of papillary thyroid cancer: Epidemiology, management, and the implications for patients. Cancer 2016;122:3754-9.

7. Nikiforov YE, Nikiforova MN. Molecular genetics and diagnosis of thyroid cancer. Nat Rev Endocrinol 2011;7:569-80.

8. Nikiforov YE, Carty SE, Chiosea SI, et al. Highly accurate diagnosis of cancer in thyroid nodules with follicular neoplasm/suspicious for a follicular neoplasm cytology by ThyroSeq v2 next-generation sequencing assay. Cancer 2014;120:3627-34. 
9. Chudova D, Wilde JI, Wang ET, et al. Molecular classification of thyroid nodules using high-dimensionality genomic data. J Clin Endocrinol Metab 2010;95:5296-304.

10. Wylie D, Beaudenon-Huibregtse S, Haynes BC, et al. Molecular classification of thyroid lesions by combined testing for miRNA gene expression and somatic gene alterations. J Pathol Clin Res 2016;2:93-103.

11. Sandler JE, Huang H, Zhao N, et al. Germline Variants in DNA Repair Genes, Diagnostic Radiation, and Risk of Thyroid Cancer. Cancer Epidemiol Biomarkers Prev 2018;27:285-94.

12. Lew JI, Solorzano CC. Use of ultrasound in the management of thyroid cancer. Oncologist 2010;15:253-8.

13. Cibas ES, Ali SZ. The 2017 Bethesda System for Reporting Thyroid Cytopathology. Thyroid 2017;27:1341-6.

14. Wu Y, Xu T, Cao X, et al. BRAF (V600E) vs. TIRADS in predicting papillary thyroid cancers in Bethesda system I, III, and V nodules. Cancer Biol Med 2019;16:131-8.

15. Zhu J, Li X, Wei X, et al. The application value of modified thyroid imaging report and data system in diagnosing medullary thyroid carcinoma. Cancer Med 2019;8:3389-400.

16. Grant EG, Tessler FN, Hoang JK, et al. Thyroid Ultrasound Reporting Lexicon: White Paper of the ACR Thyroid Imaging, Reporting and Data System (TIRADS) Committee. J Am Coll Radiol 2015;12:1272-9.

17. Tessler FN, Middleton WD, Grant EG. Thyroid Imaging Reporting and Data System (TI-RADS): A User's Guide. Radiology 2018;287:29-36.

18. Persichetti A, Di Stasio E, Guglielmi R, et al. Predictive Value of Malignancy of Thyroid Nodule Ultrasound Classification Systems: A Prospective Study. J Clin Endocrinol Metab 2018;103:1359-68.

19. Ram N, Hafeez S, Qamar S, et al. Diagnostic validity of ultrasonography in thyroid nodules. J Pak Med Assoc 2015;65:875-8.

20. Brito JP, Gionfriddo MR, Al Nofal A, et al. The accuracy of thyroid nodule ultrasound to predict thyroid cancer: systematic review and meta-analysis. J Clin Endocrinol Metab 2014;99:1253-63.

21. Chudgar AV, Shah JC. Pictorial Review of FalsePositive Results on Radioiodine Scintigrams of Patients with Differentiated Thyroid Cancer. Radiographics 2017;37:298-315.

22. Xue YL, Qiu ZL, Perotti G, et al. 131I SPECT/CT: a one-station imaging modality in the management of differentiated thyroid cancer. Clinical and Translational
Imaging 2013;1:163-73.

23. Ahmed S, Horton KM, Jeffrey RB Jr, et al. Incidental thyroid nodules on chest CT: Review of the literature and management suggestions. AJR Am J Roentgenol 2010;195:1066-71.

24. Hoang JK, Langer JE, Middleton WD, et al. Managing incidental thyroid nodules detected on imaging: white paper of the ACR Incidental Thyroid Findings Committee. J Am Coll Radiol 2015;12:143-50.

25. Abraham T, Schoder H. Thyroid cancer--indications and opportunities for positron emission tomography/ computed tomography imaging. Semin Nucl Med 2011;41:121-38.

26. Ciarallo A, Marcus C, Taghipour M, et al. Value of Fluorodeoxyglucose PET/Computed Tomography Patient Management and Outcomes in Thyroid Cancer. PET Clin 2015;10:265-78.

27. Song B, Wang H, Chen Y, et al. Magnetic resonance imaging in the prediction of aggressive histological features in papillary thyroid carcinoma. Medicine (Baltimore) 2018;97:e11279.

28. Noda Y, Kanematsu M, Goshima S, et al. MRI of the thyroid for differential diagnosis of benign thyroid nodules and papillary carcinomas. AJR Am J Roentgenol 2015;204:W332-5.

29. Yuan Y, Yue XH, Tao XF. The diagnostic value of dynamic contrast-enhanced MRI for thyroid tumors. Eur J Radiol 2012;81:3313-8.

30. Varoquaux A, Rager O, Dulguerov P, et al. Diffusionweighted and PET/MR Imaging after Radiation Therapy for Malignant Head and Neck Tumors. Radiographics 2015;35:1502-27.

31. Binse I, Poeppel TD, Ruhlmann M, et al. Imaging with (124)I in differentiated thyroid carcinoma: is PET/MRI superior to PET/CT? Eur J Nucl Med Mol Imaging 2016;43:1011-7.

32. Jentzen W, Phaosricharoen J, Gomez B, et al. Quantitative performance of (124)I PET/MR of neck lesions in thyroid cancer patients using (124)I PET/CT as reference. EJNMMI Phys 2018;5:13.

33. Jadvar H, Chen X, Cai W, et al. Radiotheranostics in Cancer Diagnosis and Management. Radiology 2018;286:388-400.

34. Budiawan H, Salavati A, Kulkarni HR, et al. Peptide receptor radionuclide therapy of treatment-refractory metastatic thyroid cancer using (90)Yttrium and (177) Lutetium labeled somatostatin analogs: toxicity, response and survival analysis. Am J Nucl Med Mol Imaging 
2013;4:39-52.

35. Santhanam P, Solnes LB, Rowe SP. Molecular imaging of advanced thyroid cancer: iodinated radiotracers and beyond. Med Oncol 2017;34:189.

36. Marcus CD, Ladam-Marcus V, Cucu C, et al. Imaging techniques to evaluate the response to treatment in oncology: current standards and perspectives. Crit Rev Oncol Hematol 2009;72:217-38.

37. Lalchandani UR, Sahai V, Hersberger K, et al. A Radiologist's Guide to Response Evaluation Criteria in Solid Tumors. Curr Probl Diagn Radiol 2019;48:576-85.

38. Gilardi L, Grana CM, Paganelli G. Evaluation of response to immunotherapy: new challenges and opportunities for PET imaging. European Journal of Nuclear Medicine and Molecular Imaging 2014;41:2090-2.

39. Kim HD, Kim BJ, Kim HS, et al. Comparison of

Cite this article as: Bonjoc KJ, Young H, Warner S, Gernon T, Maghami E, Chaudhry A. Thyroid cancer diagnosis in the era of precision imaging. J Thorac Dis 2020;12(9):5128-5139. doi: 10.21037/jtd.2019.08.37 the morphologic criteria (RECIST) and metabolic criteria (EORTC and PERCIST) in tumor response assessments: a pooled analysis. Korean J Intern Med 2019;34:608-17.

40. O JH, Lodge MA, Wahl RL. Practical PERCIST: A Simplified Guide to PET Response Criteria in Solid Tumors 1.0. Radiology 2016;280:576-84.

41. Min SJ, Jang HJ, Kim JH. Comparison of the RECIST and PERCIST criteria in solid tumors: a pooled analysis and review. Oncotarget 2016;7:27848-54.

42. O JH, Wahl RL. PERCIST in Perspective. Nucl Med Mol Imaging 2018;52:1-4.

43. Aras M, Erdil TY, Dane F, et al. Comparison of WHO, RECIST 1.1, EORTC, and PERCIST criteria in the evaluation of treatment response in malignant solid tumors. Nucl Med Commun 2016;37:9-15. 